Williams and Khan (1967) were the first to draw attention to this in one of their patients, and confirmation has come from Hoyumpa and Connell (1973) and Goldstein et al. (1973). The latter authors, in an investigation of the aetiology of 21 consecutive cases of active chronic hepatitis in their unit in 12 months, found no fewer than 14 cases which they considered to be drug induced. Of these, nine were associated with ingestion of oxyphenisatin and five methyldopa. In an analysis of the histological features they found that in active chronic hepatitis due to methyldopa there was milder liver cell necrosis and a lower incidence of cirrhosis than in active chronic hepatitis due to oxyphenisatin and active chronic hepatitis of unknown origin. They also commented on the better initial improvement in patients with chronic hepatitis who were able to stop taking their drug as compared with those in the "unknown origin" group. As with the haemolytic anaemia due to methyldopa it seems likely that the development of active chronic hepatitis has an immunological basis. Unfortunately autoantibody studies were not always reported in earlier papers and, in particular, the incidence of smooth muscle antibody is not known.

While proof of the aetiological role of methyldopa may be obtained by a rechallenge with the drug we feel that this is a highly dangerous procedure which may be followed by fatal hepatic necrosis. Some of the deaths from hepatic necrosis reported in the literature have resulted from accidental or deliberate rechallenge with the drug (Rehman et al., 1973; Hoyumpa and Connell, 1973). Though the first episode of liver damage may be delayed for several weeks after the initial treatment with methyldopa it seems clear that readministration of the drug may elicit a prompt and dramatic exacerbation of the liver injury.

In view of the high incidence of mild abnormalities in liver function after starting treatment with methyldopa we feel that there is little point in carrying out routine tests during the first few weeks of treatment. Clearly routine testing will not screen out those patients who may progress to clinical liver damage. Our experience, however, of a patient with undiagnosed cirrhosis who died from superimposed liver damage from methyldopa indicates the need for caution. It seems prudent, therefore, to check the results of routine liver function tests before starting treatment in all patients, and it is clearly dangerous to give methyldopa to patients with overt liver disease. An awareness of the possibility of methyldopa hepatotoxicity is probably the most important point and, above all, there must be a strict avoidance of the temptation to perform a clinical rechallenge, an exercise which may result in disaster.

We are grateful to Dr. W. H. W. Inman, of the Committee on Saítiy of Medicines, for his help in enabling us to contact those doctors who had reported cases of jaundice possibly due to methyldopa. The views expressed in this paper, however, are not necessarily those of the committee. We also thank the physicians, parhologists, and general practitioners who allowed us to study their cases, and we are particularly indebted to the following doctors for their help: Dr. A. Bernstein (Salford); Dr. J. A. Campbell (Norwich); Mr. R. T. Campbell (Portsmouth); Dr. T. M. Chalmers (Cambridge); Dr. J. R. Clarke (Galashiels); Dr. A. E. Dormer (Lcndon); Dr. R. Hamm (Douglas, I.O.M.); Dr. P. Harris (Wimborne); Dr. D. A. Hill (Bromley); Dr. G. F. B. Mitchell (Wakefield); Dr. S. J. S. Polwin (Portsmouth); Dr. J. G. Scott (Kendal); Dr. R. Wilkinson (Newcastle); Dr. A. K. Woodward (Wolverhamp:on).

A shortened version of this paper was presented at the spring meeting of the British Society of Gastroenterology, 1974.

\section{References}

Brouillard, R. P., and Barrett, O. (1973). Fournal of the American Medical Association, 224, 904.

Colwill, J. M., et al. (1964). New England fournal of Medicine, 271, 696

Elkington, S. G., Schreiber, W. M., and Conn, H. O. (1969). Circulation, 40,589.

Goldstein, G. B., Lam, K. C., and Mistilis, S. P. (1973). American fournal of Digestive Diseases, 18, 177.

Hoyumpa, A. M., and Connell, A. M. (1973). American fournal of Digestive Diseases, 18, 213.

Irvine, R. O. H., O'Brien, K. P., and North, J. D. K. (1962). Lancet, 1, 300 Leonard, J. W., Gifford, R. W., and Humphrey, D. C. (1965). American Heart fournal, 69, 610

Rehman, O. U., Keith, T. A., and Gall, E. A. (1973). Fournal of the American Medical Association, 224, 1390.

Sheps, S. G., et al. (1963). Fournal of the American Medical Association, 184 616.

Smith, W. M., et al. (1966). Annals of Internal Medicine, 65, 657.

Tysell, J. E., and Knauer, M. (1971). American fournal of Digestive Diseases, 16,848 .

Williams, E. R., and Khan, M. A. (1967). Fournal of Therapeutics and Clinical Research, 1, No. 6, p. 5 .

\title{
Essential Thrombocythaemia and Peripheral Gangrene
}

\author{
F. E. PRESTON， I. G. EMMANUEL， D. A. WINFIELD， R. G. MALIA
}

British Medical fournal, 1974, 3, 548-552

\section{Summary}

Six patients are described in whom gangrene of one or more toes occurred as the presenting feature of essential thrombocythaemia. Spontaneous platelet aggregation was observed in platelet-rich plasma from four patients and platelet aggregation after the addition of adenosine diphosphate and

\footnotetext{
University Department of Haematology, Royal Infirmary, Sheffield S3 3DA

F. E. PRESTON, M.D., M.R.C.PATH., Consultant/Associate Haematologist

D. A. WINFIELD, M.R.C.PATH., Senior Registrar

D. A. WINFIELD, M.R.C.PATH., Senior Registrar
R. G. MALIA, F.I.M.L.T., Senior Chief Technician

Weston Park Hospital, Sheffield S10 2SJ

I. G. EMMANUEL, F.F.R., Consultant Radiotherapist
}

collagen was highly abnormal in samples from all six. All of the patients described dramatic relief of pain within six hours of ingestion of aspirin and this coincided with disappearance of the spontaneous platelet aggregation and collagen-induced platelet aggregation. Treatment with phosphorus-32 corrected the platelet count and there were no further recurrences of peripheral vascular disease. Platelet function tests performed at the time all gave normal results. It is concluded that essential thrombocythaemia is an important and treatable cause of peripheral vascular disease.

\section{Introduction}

Though vascular thromboses are a known complication of essential thrombocythaemia it is usually major vessels which are affected (Gunz, 1960) and there have been surprisingly few reports on the importance of this condition as a cause of peripheral vascular disease. 
The six patients described here were all diagnosed as having essential thrombocythaemia while awaiting surgical intervention for gangrene of one or more toes. A causal relationship between the platelet abnormalities and the vascular lesions is afforded by the observations that striking relief from pain coincided with improved platelet function after the administration of aspirin. Moreover, recurrence of pain was temporally associated with recurrence of the abnormal platelet function.

Treatment with ${ }^{32} \mathrm{P}$ corrected the platelet counts of all six patients, who remained well and pain free. Platelet function also became normal.

\section{Patients and Methods}

The patients, all men, were investigated and treated during August 1972 to October 1973. All had impending gangrene of one or more toes at the time of diagnosis and three had already had toes amputated before the diagnosis had been suspected. In addition four of the patients had a previous history of thromboembolism involving both the arterial and venous sides of the circulation. Haematological data on the six patients are given in table I. None of the patients had abnormalities of glucose or lipid metabolism and cryoglobulins and cold agglutinins were not detected. Peripheral blood counts, performed on at least three separate occasions in all six patients, showed a persistent thrombocytosis. There were no obvious predisposing factors, and in particular there was no evidence of malignancy, chronic infection, or any other myeloproliferative disorder and none of the patients had undergone a splenectomy. Red cell mass and plasma volume were determined isotopically and were normal in all six patients. It was believed, therefore, that the patients had essential thrombocythaemia.

TABle I-Haematological Data in Patients with Essential Thrombocythaemia

\begin{tabular}{|c|c|c|c|c|c|}
\hline $\begin{array}{l}\text { Case } \\
\text { No. }\end{array}$ & $\underset{(\mathrm{g} / 100 \mathrm{ml})}{\mathrm{Hb}}$ & W.B.C. $/ \mathrm{mm}^{3}$ & Platelets $/ \mathrm{mm}^{3}$ & $\underset{(\mathrm{mm} / \mathrm{hr})}{\text { E.S.R. }}$ & $\begin{array}{l}\text { Leucocyte } \\
\text { Alkaline } \\
\text { Phosphatase } \\
\text { Score }\end{array}$ \\
\hline $\begin{array}{l}1 \\
2 \\
3 \\
4 \\
5 \\
6\end{array}$ & $\begin{array}{l}15 \cdot 6 \\
12 \cdot 2 \\
14 \cdot 9 \\
16 \cdot 4 \\
16 \cdot 2 \\
15 \cdot 6\end{array}$ & $\begin{array}{r}15,000 \\
11,000 \\
8,500 \\
7,000 \\
9,000 \\
11,500\end{array}$ & $\begin{array}{r}850,000 \\
1,250,000 \\
750,000 \\
690,000 \\
825,000 \\
750,000\end{array}$ & $\begin{array}{l}1 \\
2 \\
1 \\
2 \\
2 \\
1\end{array}$ & $\begin{array}{l}30 \\
35 \\
40 \\
28 \\
31 \\
77\end{array}$ \\
\hline
\end{tabular}

Apart from the peripheral gangrene there were no abnormal clinical features and no patient had splenomegaly.

\section{CASE 1}

A 42-year-old man was first seen by us in August 1972. He gave a six-month history of severe pain affecting the toes of both feet. A few weeks before admission to hospital he had developed some superficial ulceration of three toes of his left foot and more recently he had noticed that these had become deeply cyanosed. During the few days before admission there had been a noticeable deterioration in the general condition of his feet. Clinical examination showed apparent irreversible gangrene of the great and little toes of both feet. The other toes, though less severely affected, were blue and tender. No other abnormalities were found and all peripheral pulses were easily palpable. Results of haematological studies and platelet function tests are given in tables I and II.

The gangrenous toes were amputated and recovery was uneventful. The remaining toes were still extremely painful but aspirin $600 \mathrm{mg}$ produced dramatic relief. It was also observed that a single dose of two tablets controlled the pain for two to three days. During the postoperative period $5 \mathrm{mCi}$ of ${ }^{32} \mathrm{P}$ was administered intravenously and during the next few weeks the platelet count fell to normal levels. The platelet count remained
TABLE II-Platelet Aggregation in Essential Thrombocythaemia

\begin{tabular}{c|c|c|c|c}
\hline \multirow{2}{*}{ Case No. } & \multicolumn{2}{|c|}{\begin{tabular}{c} 
ADP-induced Aggregation \\
\cline { 2 - 5 }
\end{tabular}} & $\begin{array}{c}\text { Collagen-induced } \\
\text { Aggregation }\end{array}$ \\
\cline { 2 - 5 } & $\begin{array}{c}\text { Max. Degree } \\
(\%)\end{array}$ & $\begin{array}{c}\text { Max. Rate } \\
(\%)\end{array}$ & $\begin{array}{c}\text { Max. Degree } \\
(\%)\end{array}$ & $\begin{array}{c}\text { Max. Rate } \\
(\%)\end{array}$ \\
\hline 1 & 90 & 60 & 95 & 58 \\
3 & 100 & 79 & 100 & 82 \\
4 & 100 & 81 & 100 & 85 \\
5 & 75 & 68 & 75 & 60 \\
6 & 92 & 75 & 100 & 77 \\
\hline Normal range (n=10) & $34-87$ & $36-66$ & $52-92$ & $22-60$ \\
\hline Mean & 61 & 48 & 66 & 37 \\
\hline
\end{tabular}

normal and at the time of writing there was no evidence of enhanced platelet aggregration. The patient was well, his toes appeared healthy, and he was pain free.

\section{CASE 2}

A 45-year-old man was admitted to hospital in January 1973 for a lumbar sympathectomy. He had been in good health until six months previously, when he first presented with painful toes of the right foot. Two of these had rapidly become gangrenous and were amputated. No abnormalities were noted on arteriography and a right lumbar sympathectomy was performed in October 1972 for ischaemic pain of the remaining toes of the affected foot. Though some relief of pain was noted for about four weeks he then complained of severe pain affecting the toes of both feet. While awaiting a left lumbar sympathectomy numerous large platelets were observed on a peripheral blood film and the platelet count was found to be noticeably raised (table I). Platelet function was grossly abnormal and he was given a trial of aspirin $900 \mathrm{mg}$. Relief of pain occurred within a few hours of the first dose and after excluding other causes of peripheral vascular disease it was concluded that the symptomatology was probably a consequence of the persistently raised platelet count. A $5-\mathrm{mCi}$ dose of ${ }^{32} \mathrm{P}$ was administered intravenously and he was maintained on aspirin $300 \mathrm{mg}$ three times a day until the platelets had responded to the ${ }^{32} \mathrm{P}$. Throughout this period he was pain-free He remained well with no features of peripheral vascular disease.

CASE 3

A 55-year-old man was admitted to hospital for amputation of the great toe of his right foot. He had complained of pain affecting this and other toes of both feet for two months before admission. Apart from chronic bronchitis he had been in reasonably good health until the age of 52 . Since then he had been admitted to hospital on two occasions for deep vein thrombosis and myocardial infarction complicated by cerebral embolism. After the second admission he had been maintained on oral anticoagulants. Before amputation arteriography had shown no arterial obstruction and all peripheral pulses were readily palpable. Immediately before operation abnormally large platelets were noted on the peripheral blood film and the platelet count was $750,000 / \mathrm{mm}^{3}$. Platelet function, studied at the same time, was also abnormal (see table II). After amputation the platelet count remained persistently raised and platelet function, which was retested four weeks later, was still abnormal. After further investigations (see table I) it was concluded that he had essential thrombocythaemia. Aspirin relieved the pain and this coincided with return of the platelet function to normal.

The patient was given $4 \mathrm{mCi}$ of ${ }^{32} \mathrm{P}$ and the platelet count fell to normal. Aspirin was withdrawn. There was no recurrence of pain and no further evidence of peripheral vascular disease was noted. He no longer received oral anticoagulants.

CASE 4

A 52-year-old Polish steelworker was admitted to hospital in March 1973 for a right lumbar sympathectomy. He had had two 
gangrenous toes of his right foot amputated four months previously but since then he had complained of increasing pain of the remaining toes of that foot and also of two toes of the left foot. Arteriography had shown no abnormality and all peripheral pulses were palpable. For 18 months he had attended his general practitioner for transient blurring of vision of both eyes. A precise diagnosis had not been made but there was no abnormality, apart from the peripheral gangrene, of the cardiovascular system.

Before the lumbar sympathectomy was performed morpholcgically abnormal platelets were noted on the peripheral blood film and the platelet count was found to be $690,000 \mathrm{~mm}^{3}$. After the sympathectomy there was some lessening of the pain but this began to recur five weeks later. Reinvestigation showed abnormal platelet function and persistent thrombocytosis (tables I and II) and when taken in conjunction with other clinical and laboratory data a diagnosis of essential thrombocythaemia was made. Dramatic relief of pain occurred after the introduction of aspirin and the platelet count returned to normal after $4 \mathrm{mCi}$ ${ }^{32} \mathrm{P}$ had been given intravenously. At that stage the aspirin was withdrawn. There was no recurrence of pain and the patient remained symptom free. Moreover, no further visual disturbances occurred.

CASE 5

A 59-year-old man was admitted to hospital in June 1973 for amputation of a pregangrenous fourth left toe. For two months he had complained of severe pain of the affected toe. and this had become cyanosed and ulcerated in the two weeks before admission. Arteriography had shown nothing of note and on clinical examination all peripheral pulses were palpable.

The patient had been well until 1971, when he had two separate, radiologically confirmed, deep vein thromboses which had been treated with anticoagulants. In 1972 he had a myocardial infarction and since then had been taking oral anticoagulants.

While awaiting amputation numerous large platelets were noted on the peripheral blood film and the platelet count was found to be raised $\left(825,000 / \mathrm{mm}^{3}\right)$. Further investigation; wer performed (tables I and II) and other causes of peripheral vascular disease and secondary thrombocythaemia were excluded. It was concluded that the diagnosis was essential thrombocythaemia and after discontinuing the anticoagulants the patient was given aspirin and $4 \mathrm{mCi}$ of ${ }^{32} \mathrm{P}$. The clinical response to aspirin was particularly dramatic and four hours after taking the first $600 \mathrm{mg}$ he was pain free and able to walk around the ward. During the next few days the colour of the affected toe improved and amputation was deemed unnecessary. During the next few weeks the platelet count gradually fell to normal, aspirin was withdrawn, and two weeks later the platelet function was found to be normal. No further treatment was given and the patient remained symptom free without evidence of peripheral vascular disease.

CASE 6

A 61-year-old man was admitted to hospital in October 1973 for amputation of a painful gangrenous third left toe. During the previous year he had complained of episodes of transient blurring of vision but no cause for this had been discovered. In March 1973 he first complained of severe pain of the toes of both feet and in April a gangrenous second left toe was amputated. Further severe pain resulted in a left lumbar sympathectomy being performed but this gave only temporary relief and within five weeks he again complained of severe pain. Arteriography was of no diagnostic value and all peripheral pulses were palpable.

Preoperatively it was noted that the platelet count was high but in view of the condition of the affected toe this was amputated. Three weeks later the patient again complained of painful toes and again the raised platelet count was noted. After extensive investigation a diagnosis of essential thrombocythaemia was made and he was given aspirin and $5 \mathrm{mCi}$ of ${ }^{32} \mathrm{P}$. Again there was dramatic relief of pain after the ingestion of two aspirin tablets and the platelet funotion returned to normal. Aspirin was continued until the platelet count returned to normal. The patient remained symptom free and his other toes appeared healthy. No further visual disturbances occurred.

\section{PLATELET FUNCTION}

Platelet-rich plasma was prepared from venous blood collected into siliconized tubes using $1 / 10$ volume $(3.8 \% \mathrm{w} / \mathrm{v})$ sodium citrate as anticoagulant. Specimens were centrifuged at $600 \mathrm{~g}$ for five minutes. The platelet-rich plasma was diluted with the patient's own platelet-poor plasma to give a final concentration of $250,000 / \mathrm{mm}^{3}$. Plasma samples were kept at room temperature in siliconized tubes and tested 60 minutes after venesection. Aggregation studies were performed using an E.E.L. aggregometer and a Telsec flat-bed recorder.

The aggregating reagents used were adenosine diphosphate (ADP) $2.5 \mu \mathrm{g} / \mathrm{ml}$ ) and bovine tendo Achillis collagen (Sigma Chemical Co.). The terms used to describe the platelet aggregation (table II) are those described by Thomson et al. (1973).

Spontaneous platelet aggregation was detected by continuous stirring of the diluted platelet-rich plasma for three to five minutes.

\section{Results}

Evidence of spontaneous platelet aggregation was sought in samples of platelet-rich plasma from five patients. Aggregation occurred in samples examined from four patients and began after three to 10 minutes of continuous stirring. Spontaneous platelet aggregation did not occur in any specimens obtained from 15 normal subjects.

Results of platelet aggregation after the addition of ADP and collagen are given in table II. All six patients showed enhanced aggregation to both reagents.

\section{PLATELET FUNCTION AFTER ASPIRIN INGESTION}

Spontaneous Platelet Aggregation.-Specimens tested six hours after aspirin $(600 \mathrm{mg})$ ingestion showed no evidence of spontaneous platelet aggregation (fig. 1). This correction of platelet function persisted for about 72 hours after the single administration of aspirin. It was again reversed by further treatment and spontaneous platelet aggregation was not observed while the patients were maintained on $300 \mathrm{mg}$ aspirin twice daily.

Collagen-induced Platelet Aggregation.-Marked impairment of the collagen-induced response was observed a few hours after the administration of aspirin $600 \mathrm{mg}$ (fig. 2). The effect was noted in samples of platelet-rich plasma from nor$\mathrm{mal}$ and thrombocythaemic subjects and persisted for a few days after the ingestion of a single dose of aspirin.

\section{PLATELET AGGREGATION AFTER ${ }^{32} \mathrm{P}$}

In all six patients platelet counts fell to normal levels within a few weeks of the intravenous administration of ${ }^{32} \mathrm{P}$. Plate-

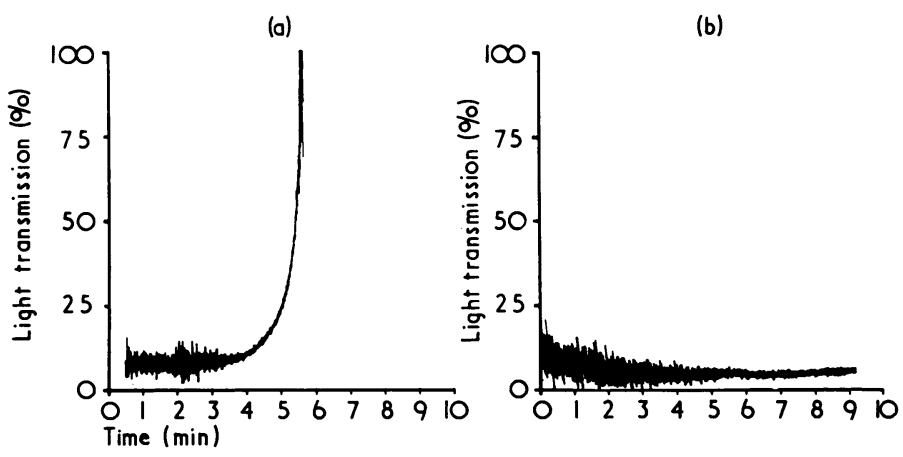

FIG. 1-Case 3. Spontaneous platelet aggregation (a) before aspirin and (b) after aspirin $600 \mathrm{mg}$. 

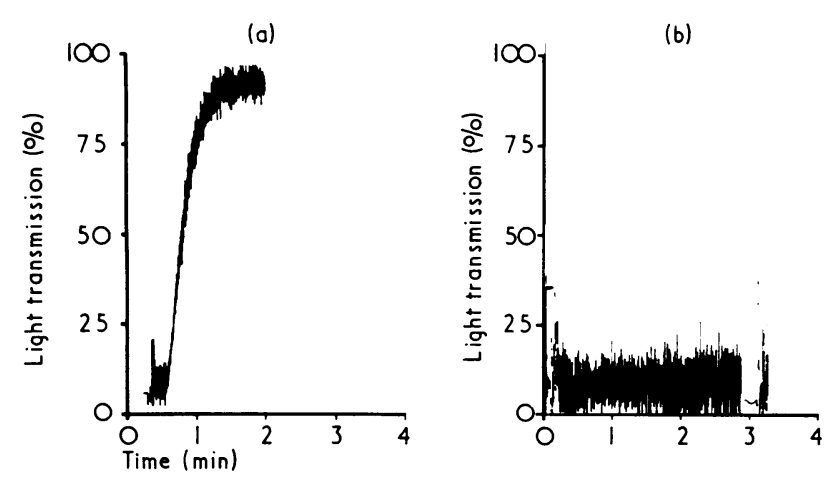

FIG. 2-Case 3. Collagen-induced platelet aggregation (a) before aspirin and (b) after aspirin $600 \mathrm{mg}$.

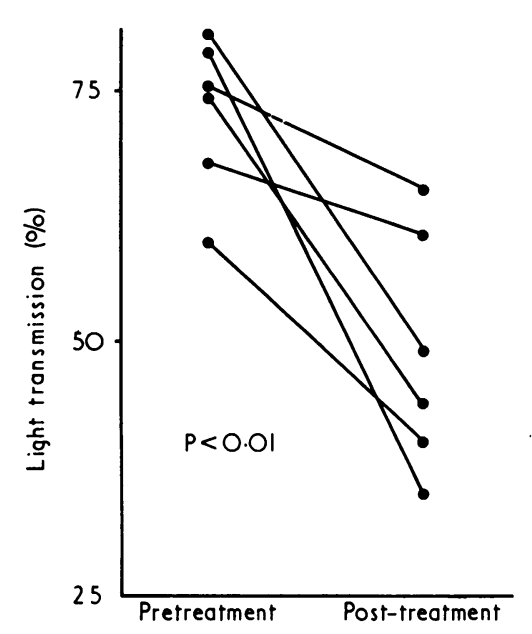

FIG. 3-Maximum rate of ADP-induced platelet aggregation in the six patients before and after ${ }^{32} \mathrm{p}$.

let aggregation, retested when the platelet count was normal, was vastly improved (fig. 3) and there was no evidence of spontaneous platelet aggregation.

\section{Discussion}

Persistent thrombocytosis may occur in association with carcinoma or as a consequence of splenectomy. It is also a recognized feature of certain myeloproliferative disorders, notably polycythaemia vera, myelofibrosis, and chronic myeloid leukaemia (Fountain and Losowsky, 1962). Thrombocytosis can however, occur as an isolated but persistent abnormality and it is now generally believed that this probably represents another example of a myeloproliferative disorder, designated essential thrombocythaemia.

It may be argued that the diagnosis of essential thrombocythaemia is not strictly tenable since the platelet counts quoted here are lower than one normally associates with this condition. We feel, however, that the diagnosis is justified because our patients had persistent thrombocytosis but without an apparent predisposing cause. Subdividing patients on the basis of relative degrees of thrombocytosis does not appear to offer any great advantage and we feel that the creation of any further distinction would be somewhat artificial.

It is possible, of course, that the observed thrombocytosis occurred as a consequence of the peripheral vascular disease. We believe that this is highly unlikely, since correction of the platelet count of ${ }^{32} \mathrm{P}$ would not in that case reverse the vascular lesion. Moreover, we have recently studied other patients with peripheral vascular disease, and thrombocytosis does not appear to be the usual response to this disorder.

It is of interest that none of our patients had any evidence of a bleeding tendency, which is a frequent complication of myeloproliferative thrombocythaemia (Hardisty and Wolff, 1955; Gunz, 1960; Fountain and Losowsky, 1962). In this context only one patient had a platelet count greater than 1 $\times 10^{6} / \mathrm{mm}^{3}$. Most of the previously described patients with haemorrhagic thrombocythaemia had platelet counts well in excess of $1 \times 10^{6} / \mathrm{mm}^{3}$ (Ozer et al., 1960; Fountain and Losowsky, 1962) and it may be that patients with essential thrombocythaemia and a relatively low platelet count are more likely to develop vascular thromboses than abnormal haemorrhage.

Thrombotic episodes are well known complications of essential thrombocythaemia but most workers have laid emphasis on the involvement of the larger vessels, particularly thrombosis of the splenic vein, and there have been only sporadic reports of peripheral gangrene as a presenting feature of this condition. Moreover, though attempts have been made to determine the cause of the abnormal bleeding in essential thrombocythaemia there have been few reports of abnormal platelet function in the thrombotic variant of this disorder.

The platelets of all our six patients showed marked enhancement of aggregation to ADP and collagen. Of particular interest was the spontaneous platelet aggregation which was observed in platelet-rich plasma from four of the five patients in whom this investigation was undertaken. This abnormality has been previously described in a patient with idiopathic thrombosis and recurrent painful toes and fingers (Vreeken and Van Aken, 1971). Examination of the data in that report suggests that the patient had essential thrombocythaemia, and again the initial platelet count was less than $1 \times 10^{4} / \mathrm{mm}^{3}$.

The phenomenon of spontaneous platelet aggregation could possibly have been explained by the presence of ADP in the patient's plasma. This was excluded by suspending normal platelets in the affected patient's plasma and then repeating the investigation. Platelet aggregation was not observed. Conversely the spontaneous aggregation could be reproduced by resuspending the patient's platelets in normal plasma.

All of our patients complained of severe pain affecting one or more toes. The administration of aspirin $600-900 \mathrm{mg}$ was followed by rapid and striking relief of pain. This response was reported by Biermé et al. (1972) and we believe that it may be a useful diagnostic test of thrombocythaemic-induced gangrene.

Coincident with the positive clinical response the ingestion of aspirin was associated in all six patients with marked impairment of collagen-induced platelet aggregation. This effect has been described by a number of workers (Mills, 1972) and it is possible that it is mediated via the incorporation of the acetyl radical into the platelet membrane (Youssef and Barkhan, 1969). A previously observed but less well recognized response to aspirin was the disappearance of the spontaneous platelet aggregation (Vreeken and Van Aken, 1971). The correction persisted for a few days after a single dose of aspirin and was thus unrelated to the clearance of the drug from the circulation. In this respect the induced changes are similar to the impaired collagen response.

Treatment with ${ }^{32} \mathrm{P}$ effected a satisfactory response in all patients and platelet counts fell to normal levels within a few weeks. At this time the patients were asymptomatic and the initial platelet abnormalities were no longer present.

It appears from our findings that the peripheral gangrene originally present in the six patients described here was a direct consequence of the thrombocytosis and enhanced platelet reactivity. We believe also that the incidence of thrombotic thrombocythaemia is greater than is generally believed, and since treatment is simple and effective a plate- 
let count should be performed in all patients with peripheral vascular disease.

We are grateful for financial suppont for some of this work from the Endowment Research Fund of the United Sheffield Hospitals.

\section{Addendum}

Since preparing this report two similar cases (one man and one woman) have been investigated and treated by us.

\section{References}

Biermé, R., et al. (1972). Lancet, 1, 432.
Fountain, J. R., and Losowsky, M. S. (1962). Quarterly fournal of Medicine, 31, 207.

Gunz, F. W. (1960). Blood, 15, 706.

Hardisty, R. M., and Wolff, H. H. (1955). British fournal of Haematology, 1, 390.

Lewis, S. M., Szur, L., and Hoffbrand, A. V. (1972). Clinics in Haematology, $1,339$.

Mills, D. C. B. (1972). Clinics in Haematology, 1, 295.

Ozer, F. L., et al. (1960). American fournal of Medicine, 28, 807.

Thomson, C., Forbes, C. D., and Prentice, C. R. M. (1973). Clinical Science and Molecular Medicine, 45, 485.

Vreeken, J., and Van Aken, W. G. (1971). Lancet, 2, 1394.

Youssef, A. H., and Barkhan, P. (1969). British Medical fournal, 3, 394.

\title{
Deaths from Occlusive Arterial Disease in Renal Allograft Recipients
}

\author{
L. S. IBELS, J. H. STEWART, J. F. MAHONY, A. G. R. SHEIL
}

British Medical fournal, 1974, 3, 552-554

\section{Summary}

In a series of 325 recipients of cadaveric renal transplants sudden occlusive arterial disease was found to be responsible for $12 \%$ of deaths. Acute myocardial infarction $(9 \%)$ occurred 25 times more than expected in the normal population and cerebral thrombosis $(3 \%) 300$ times more. The greatest loss was in the initial three-month period after transplantation. Patients with renal failure due to essential hypertension were especially at risk, accounting for six of the 12 deaths.

\section{Introduction}

The apparently high incidence of fatal arterial occlusive disease in renal allograft recipients (Australian National Renal Transplantation Survey, 1971; Advisory Committee to the Renal Transplant Registry, 1972; Brunner et al., 1972; Lowrie et al., 1973) suggests a higher mortality from these causes than in the normal population.

To determine the accuracy of this observation the mortality due to acute coronary and cerebral arterial thrombosis was determined in a consecutive series of 325 patients who received cadaveric renal transplants during a six-year period.

\section{Patients and Methods}

Between August 1967 and May 1973349 cadaveric renal transplant operations were carried out on 325 recipients at Sydney Hospital and the Royal Prince Alfred Hospital (Sheil et al., 1972, 1973). The total follow-up experience to May 1973 was 570 patient years, during which time there were 100 deaths. The causes of death were determined from necropsy and hospital clinical records.

\footnotetext{
Medical Research Department, Kanematsu Memorial Institute, Sydney Hospital, Sydney, Australia

L. S. IBELS, M.B., M.R.A.C.P., Senior Registrar, Immunology and Renal Unit J. H. STEWART, M.R.C.P., M.R.A.C.P., Associate Director

J. F. MAHONY, M.B., F.R.A.C.P., Physician, Immunology and Renal Unit

Department of Surgery, University of Sydney, Sydney, Australia A. G. R. SHEIL, F.R.C.S., F.R.A.C.S., Associate Professor
}

Age- and sex-specific death rates in Australia for acute myocardial infarction and cerebral thrombosis (respectively 410 and 433 in the international classification of causes of death) obtained from published data of the Commonwealth Bureau of Census and Statistics (O'Neill, 1971) were used to derive the expected mortality from these causes in the number of person years at risk in the transplant population. Since the probability of death from either of these causes is small statistical analysis was based on a Poisson approximation.

\section{Results}

Of the 100 deaths in this transplant population nine were certainly and two possibly due to acute coronary occlusion, and three resulted from cerebral thrombosis (table I). Necropsy confirmation was obtained in all three with cerebral thrombosis and in seven of the patients with coronary thrombosis. In the two other certain cases myocardial infarction was proven by serial electrocardiography and serum enzyme determinations after their admission to hospital with a clinical history suggestive of this diagnosis. Death was possibly due to coronary artery occlusion in two patients who died suddenly at home when in apparently good health (one had had severe atherosclerosis).

Seven deaths were due to cerebral haemorrhage. Three of these occurred in patients on anticoagulant therapy, and one was due to a ruptured cerebral aneurysm. In this study these seven deaths were excluded from further consideration.

Occlusive vascular mortality was greatest in the early posttransplantation period, and five deaths occurred in the first month and three others in the next two months after operation. Five of the nine patients dying from proven coronary artery occlusion had had ischaemic heart disease before transplantation. Graft function at the time of acute arterial occlusion was good in all but two patients (cases 9 and 14). In one further patient (case 4) poor graft function at the time of his death was due to prolonged hypotension resulting from his myocardial infarction.

The calculated expected mortality in this transplant population was 0.36 death from myocardial infarction and 0.01 from cerebral thrombosis. The observed mortalities-nine and three deaths-were respectively 25 times and 300 times greater, both highly significant increases.

When the renal allograft recipients were grouped according to their original renal disease those with essential hypertension had the highest overall death rate (table II). This was due to a mortality from occlusive arterial disease of $26 \%$, which was significantly higher than that of the patients in any of the other diagnostic categories except those with polycystic renal disease. 